\title{
Stage IIC Testicular Cancer AJCC v6 and v7
}

National Cancer Institute

\section{Source}

National Cancer Institute. Stage IIC Testicular Cancer A/CC v6 and v7. NCI Thesaurus.

Code C6366.

Stage IIC includes: (Any pT/TX, N3, M0, S0); (Any pT/TX, N3, M0, S1). pTX: Primary tumor cannot be assessed. N3: Metastasis with a lymph node mass more than $5 \mathrm{~cm}$ in greatest dimension. M0: No distant metastasis. S0: Marker study levels within normal limits. S1:

LDH less than $1.5 \times \mathrm{N}$ ( $\mathrm{N}$ indicates the upper limit of normal for the LDH assay) and hCG less than 5,000 and AFP less than 1,000. (AJCC 6th and 7th eds.) 